\title{
SURF IA CONFLICT DETECTION AND RESOLUTION ALGORITHM EVALUATION
}

\author{
Denise R. Jones, Ryan C. Chartrand, Sara R. Wilson, and Sean A. Commo \\ National Aeronautics and Space Administration, Hampton, VA \\ Sharon D. Otero and Glover D. Barker, Lockheed Martin, Hampton, VA
}

\begin{abstract}
The Enhanced Traffic Situational Awareness on the Airport Surface with Indications and Alerts (SURF IA) algorithm was evaluated in a fast-time batch simulation study at the National Aeronautics and Space Administration (NASA) Langley Research Center. SURF IA is designed to increase flight crew situation awareness of the runway environment and facilitate an appropriate and timely response to potential conflict situations. The purpose of the study was to evaluate the performance of the SURF IA algorithm under various runway scenarios, multiple levels of conflict detection and resolution (CD\&R) system equipage, and various levels of horizontal position accuracy. This paper gives an overview of the SURF IA concept, simulation study, and results.
\end{abstract}

\section{Introduction}

Runway incursions are a serious aviation safety hazard. As such, the FAA is committed to reducing the severity, number, and rate of runway incursions by implementing a combination of guidance, education, outreach, training, technology, infrastructure, and risk identification and mitigation initiatives [1]. Progress has been made in reducing the number of serious incursions - from a high of 67 in Fiscal Year (FY) 2000 to 6 in FY2010. However, the rate of all incursions has risen steadily over recent years - from a rate of 12.3 incursions per million operations in FY2005 to a rate of 18.9 incursions per million operations in FY2010 [1, 2]. The National Transportation Safety Board (NTSB) also considers runway incursions to be a serious aviation safety hazard, listing runway incursion prevention as one of their most wanted transportation safety improvements [3]. The NTSB recommends that immediate warning of probable collisions/incursions be given directly to flight crews in the cockpit [4].

The Enhanced Traffic Situational Awareness on the Airport Surface with Indications and Alerts
(SURF IA) application has been established by RTCA Special Committee 186 to reduce the likelihood and severity of runway incursions and collisions. Safety, performance, and interoperability requirements (SPR) [5] have been developed for SURF IA to increase flight crew situation awareness of the runway environment and facilitate an appropriate and timely response to potential conflict situations. The SURF IA application utilizes cockpit display of traffic information (CDTI) to promote surface situation awareness and associated flight deck indication and alerting concepts for safety assurance. The application employs continual own-ship and traffic data monitoring and algorithms to detect potential conflicts on the runway. Several human-inthe-loop studies have been conducted to evaluate the SURF IA concept [6, 7, and 8].

A fast-time batch simulation study was conducted to evaluate the performance of the SURF IA algorithm, with variations in surveillance accuracy. The algorithm was evaluated under various runway scenarios, multiple levels of $C D \& R$ system equipage, and various levels of horizontal position accuracy. Algorithm performance was assessed through various metrics including the frequency of collisions and near collisions and nuisance and missed alerts. This paper presents an overview of the SURF IA concept, description of the test method, and study results.

\section{System Description}

\section{Simulation Tool}

A simulation tool, known as Traffic Manager (TMX), was utilized for this study. TMX is a desktop simulation application designed for interaction studies of aircraft in present or future Air Traffic Management environments [9]. TMX can serve as a stand-alone traffic simulator, scenario generator, scenario editor, experiment control station, data 
recording tool, and rapid prototyping environment and can operate in real-time or fast-time mode. For this study, TMX was used in fast-time mode simulating various approach, departure, and taxi scenarios at the Chicago O'Hare International (KORD) airport. Although TMX is capable of simulating up to 2,000 aircraft simultaneously, only two aircraft per scenario were simulated. Each aircraft used a six-degree-of-freedom dynamics model.

Some modifications were made to TMX for this study. These included: 1) an updated database for the KORD airport; 2) creation of a Global Positioning System (GPS) sensor model for position accuracy; 3) creation of an interface to the CD\&R algorithm; 4) expansion of the pilot model to handle the required taxi and runway maneuvers; and various other minor modifications.

\section{Surveillance Data}

Traffic data are integral to the SURF IA concept. For this study, it was assumed that Automatic Dependent Surveillance - Broadcast (ADS-B) would be used as the means for transmitting (ADS-B Out) and receiving (ADS-B In) aircraft surveillance data. ADS-B transmissions followed RTCA DO-242A specifications [10]; however, some simplifications were made to minimize computational overhead, such as not implementing models for latency effects and transmission line-of-sight and bandwidth blockage. For state-vector messages, a $1 \mathrm{~Hz}$ data transmission rate was specified. The position accuracy depended on the GPS measurement errors including a bias and an instantaneous jitter. A GaussMarkov process was used to model the time correlation between successive position measurement errors [11].

Navigation Accuracy Category for Position (NACp) describes the accuracy of positional information. NACp values range from 0 to 11 [10]. The horizontal Estimated Position Uncertainty (EPU) values for NACp categories of 8 and higher are listed in Table 1.

The Federal Aviation Administration (FAA) has issued an ADS-B Out Final Rule [12] which includes performance standards for ADS-B Out. The rule states that EPU must be less than 0.05 nautical miles $(\mathrm{nm})$, which is equivalent to NACp 8.
Table 1. NACp

\begin{tabular}{|c|c|}
\hline NACp & $95 \%$ Horizontal Accuracy Bound (EPU) \\
\hline 8 & EPU $<92.6 \mathrm{~m}(0.05 \mathrm{~nm}, 305.6 \mathrm{ft})$ \\
\hline 9 & EPU $<30 \mathrm{~m}(99 \mathrm{ft})$ \\
\hline 10 & EPU $<10 \mathrm{~m}(33 \mathrm{ft})$ \\
\hline 11 & EPU $<3 \mathrm{~m}(9.9 \mathrm{ft})$ \\
\hline
\end{tabular}

Irrespective of this rule, the SURF IA SPR [5] has proposed horizontal position accuracy requirements. Through analysis, the SPR identified that to meet safety requirements, horizontal position accuracy on the airport surface needs to be at least 10 $\mathrm{m}$ within $95 \%$ containment bounds (NACp 10) to allow indications and alerts at virtually all airports in the National Airspace System. Validation of these requirements is on-going.

Based on the ADS-B Out Final Rule and SURF IA requirements, NACp values of $8,9,10$, and 11 were evaluated for this study. Truth data, with no accuracy errors, was also evaluated.

\section{Conflict Detection}

\section{SURF IA Algorithm}

SURF IA identifies potential runway conflicts that involve aircraft or vehicles in the airport maneuvering area and within $3 \mathrm{~nm}$ of the runway threshold and $1000 \mathrm{ft}$ above field elevation (AFE). SURF IA generates both indications and alerts for a Cockpit Display of Traffic Information (CDTI). SURF IA utilizes traffic surveillance information obtained from ADSB-In and generates indications and alerts based on the aircraft/vehicle states during same runway, very closely spaced parallel runway, and intersecting runway operating configurations. Six types of aircraft operational states are defined: 1) taxiing on a taxiway toward a hold line or stopped at a hold line; 2) entering or crossing a runway (not lined up with runway); 3) takeoff; 4) approach; 5) after landing roll-out on runway (e.g., less than or equal to $40 \mathrm{kt}$ ); and 6) stopped or taxiing along a runway. To prevent inappropriate crew responses during departure, indications and alerts are inhibited above $80 \mathrm{kt}$. The SURF IA application does not currently address taxiway or low altitude air-to-air conflicts, directive alerting, and is not intended for use on helicopters or vehicles. A complete description of the SURF IA application can be found in [5]. 


\section{Indications and Alerts}

Indications and alerts notify the flight crew of potentially hazardous situations and are presented to the flight crew on a CDTI [5].

Indications are intended to generate pilot awareness and situation assessment by highlighting the runway and traffic status as relevant to own-ship operations. Indications identify operational conditions that are generally normal, yet relevant for runway safety and could be a precursor to a nonnormal situation. Two types of indications are defined.

A traffic indication (TI) highlights a potential runway traffic collision/hazard that may emerge in the near future. TIs are intended to increase the flight crews' awareness of the relevant runway traffic. After a brief assessment of the situation and if appropriately cleared, the flight crew may proceed with the intended operation.

A runway status indication (RSI) identifies if the runway that own-ship is approaching or using is inuse or occupied by other traffic and is not suitable for entering, takeoff, or landing. Before proceeding, the crew should ensure they have the appropriate clearance and the indicated traffic is not a factor.

Alerts identify potential collision hazards which may require timely response by the flight crew to avoid a collision. Alerts have priority over indications. Auditory and visual annunciations are required for alerts. A two-level alerting scheme is defined.

Caution alerts are generated for conditions that require immediate flight crew awareness and subsequent flight crew response. Generally, caution alerts are generated with sufficient time to evaluate the situation to be prepared to respond.

Warning alerts are generated for conditions that require immediate flight crew awareness and immediate flight crew response. Warning alerts may occur without preceding caution alerts.

\section{Test Method}

Data collection occurred for runway conflict scenarios. Only two aircraft were included in each scenario to limit the interaction in this initial fasttime study. For ease of discussion, the aircraft will be referred to as Aircraft A and Aircraft B.

\section{CD\&R Equipage}

Various levels of CD\&R system equipage were simulated for this study: a) both aircraft equipped; b) neither aircraft equipped; or, c) one or the other aircraft equipped.

When an aircraft was not equipped, it would follow its planned flight path to the end of the test run. When an aircraft was equipped, it would take action after a warning alert was generated by following an appropriate maneuver (e.g. go-around, abort, stop), depending on the operational phase. The maneuver was based on the relative location of the aircraft at the projected closest point of approach (CPA).

\section{Test Scenarios}

Seven runway scenarios were developed. Variability was introduced into the scenarios by varying the location of the aircraft, speed of the aircraft, and/or time when the aircraft started to proceed along its predefined route. As a result, not every test run resulted in a conflict or collision. Due to space limitations, only two scenarios are reported in this document.

\section{Runway Scenario - Arrival with taxi crossing}

This scenario evaluated the situation where an aircraft was on approach when another aircraft taxied perpendicular across the runway.

The initial condition (IC) for Aircraft A was at $3.5 \mathrm{~nm}$ from the threshold at $1110 \mathrm{ft}$ above field level (AFL) at an indicated airspeed of $138 \mathrm{kt}$. Aircraft A flew at a constant altitude to intercept the glideslope at $3.3 \mathrm{~nm}$ from the threshold and then descended on a 3 degree glidepath for a straight-in approach to Runway 10. For the nominal flight plan, Aircraft A landed, decelerated at $2.5 \mathrm{~m} / \mathrm{s}$, taxied down the runway at $30 \mathrm{kt}$, and then slowed to exit the runway at Taxiway M7 at $3 \mathrm{kt}$. Aircraft B started at various locations around Runway 10 and taxied across starting from a complete stop and accelerating at 1 $\mathrm{m} / \mathrm{s} / \mathrm{s}$ to $15 \mathrm{kt}$. The actual taxiways for KORD were not used in this study. Instead, Aircraft B's initial position was placed at 14 different locations along the length of Runway $10(0,1000,1500,2000,2500$, $3000,3500,4000,4500,5000,6000,7500,9000$, and 10,000 feet from the runway threshold) simulating various taxiway entry points and at 18 locations away from the runway $(300,320,340,360,380,400,450$, 
$500,550,600,650,700,800,900,1,000,1,200$, 1,400 , and 1,600 feet from the runway centerline) see Figure 1. Aircraft B (red chevron, Figure 1) began to taxi when Aircraft A (blue chevron, Figure 1) was at various points in its approach and rollout (from $3.5 \mathrm{~nm}$ to the glidepath intercept on the runway, at $0.5 \mathrm{~nm}$ intervals, crossing the runway threshold, glidepath aimpoint (1000 ft), and $3000 \mathrm{ft}$, $4500 \mathrm{ft}, 1 \mathrm{~nm}, 8000 \mathrm{ft}$, and $9000 \mathrm{ft}$ past the threshold). If maneuvering were required based on a $C D \& R$ warning alert being triggered in an appropriately equipped aircraft, Aircraft A would conduct a go-around if above $70 \mathrm{ft}$ AFL; otherwise, it would continue to land and stop on the runway. Aircraft B, if equipped, would stop if its nose had not reached the runway shoulder (greater than $100 \mathrm{ft}$ from the runway centerline) at the projected stopping point; otherwise, it would continue to taxi across the runway. When conducting an emergency stop by either aircraft, a $4 \mathrm{~m} / \mathrm{s} / \mathrm{s}$ deceleration rate was used.

\section{Runway Scenario - Departure with taxi crossing}

This scenario tested the situation where an aircraft was on departure when another aircraft taxied perpendicular across the runway.

The configuration for this scenario was similar to the arrival with taxi crossing scenario, except the IC for Aircraft A was in position on Runway 10 for departure. Aircraft B's IC was placed at 12 different locations along the length of Runway $10(0,60,280,660,1,100,1,800,2,500,3,400,5,200$, $6,800,8,000$, and 9,000 feet from the runway threshold) and at the same 18 locations away from the runway as in the previous scenario - see Figure 2. Aircraft B began to taxi when Aircraft A was at various speeds and locations along its departure and climb out $(0,20,40,60,80,100,120$, and $140 \mathrm{kt}$, liftoff, and 100, 300, and $500 \mathrm{ft}$ AFL). If maneuvering were required based on a warning alert being triggered in an appropriately equipped aircraft, Aircraft A would abort departure if below takeoff decision speed $(131 \mathrm{kt})$ and stop on the runway; otherwise it would continue departure. Aircraft B, if equipped, would maneuver as described in the previous scenario.

\section{Pilot Reaction Delay}

A delay was incorporated to simulate the reaction time from when a pilot would receive a warning alert until action was taken to resolve the situation. The following delay times were used for this study: 5 seconds (sec) when aircraft was on approach, $3 \mathrm{sec}$ when aircraft was rolling out, $2 \mathrm{sec}$ during taxi, and $2 \mathrm{sec}$ during departure. These delay times were selected based on reaction delays experienced during previous piloted simulation and flight testing.

\section{Test Matrix}

Algorithm performance was evaluated for the conflict scenarios described above using the SURF IA CD\&R algorithm for five levels of surveillance accuracy (NACp of 8, 9, 10, 11, and truth), and four levels of $C D \& R$ system equipage (neither aircraft equipped, only Aircraft A equipped, only Aircraft B equipped, and both aircraft equipped). Thus, 20 cases were examined for each of the scenarios.

The number of replicates for each treatment combination varied according to level of surveillance accuracy. As shown in Table 2, more replicates were conducted for the lower NACp values in order to increase the level of precision for estimating the true location of the aircraft.

\section{Table 2. Number of Replicates}

\begin{tabular}{|c|c|}
\hline NACp & Number of replicates \\
\hline 8 & 7 \\
\hline 9 & 6 \\
\hline 10 & 4 \\
\hline 11 & 3 \\
\hline Truth & 1 \\
\hline
\end{tabular}

Some of the test conditions from Figures 1 and 2 , in which a conflict would obviously not occur, were omitted in order to reduce the size of the test matrix.

Data were collected for the test runs in random order by scenario. For data manageability of the scenarios that required a large number of test runs, data were collected randomly grouped by level of surveillance accuracy and CD\&R system equipage.

\section{Test Metrics}

Some of the metrics utilized for this study are defined in this section. All data is referenced from the aircraft center-of-gravity (CG), unless noted otherwise. 


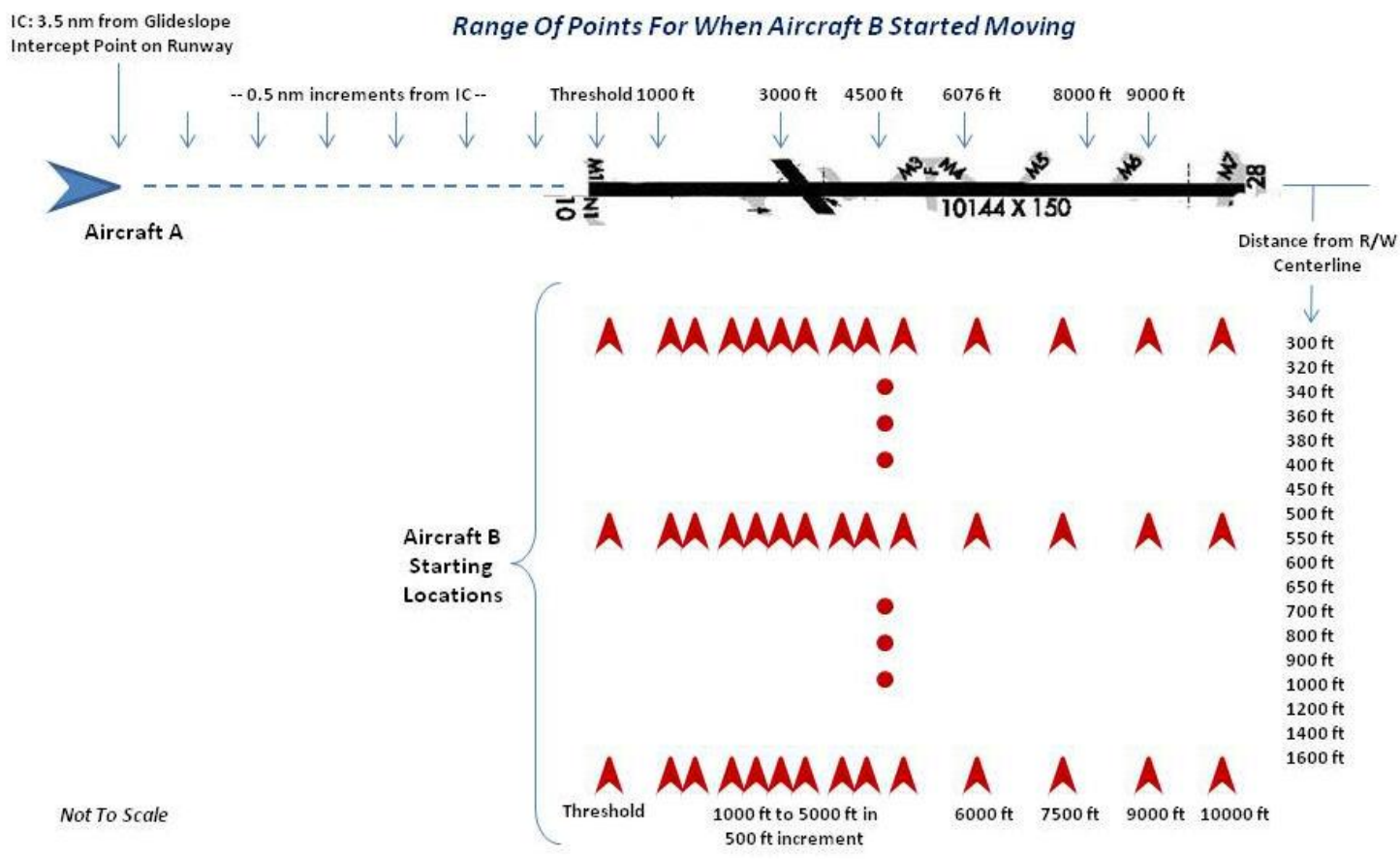

Figure 1. Runway Scenario - Arrival with Taxi Crossing Initial Conditions

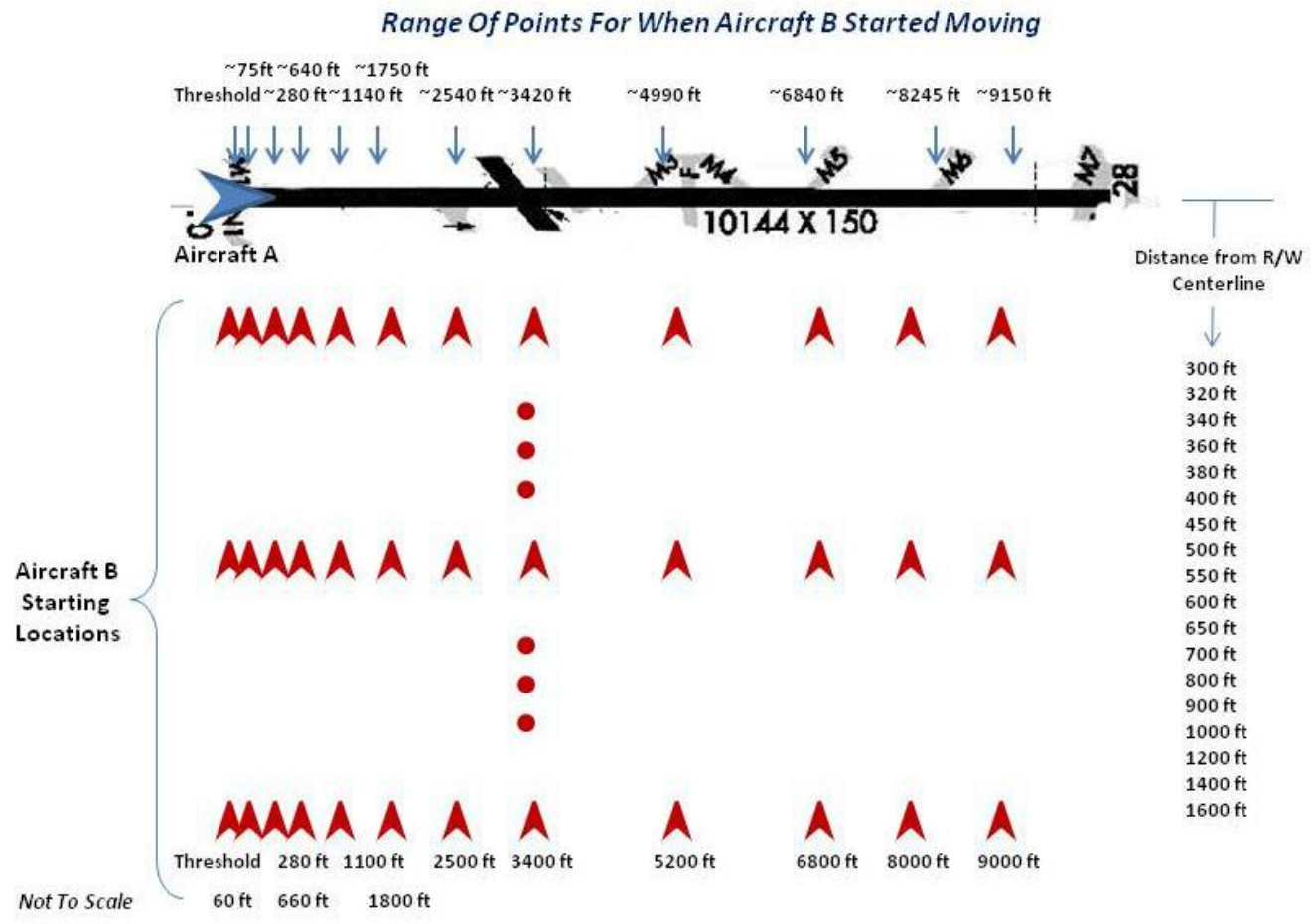

Figure 2. Runway Scenario - Departure with Taxi Crossing Initial Conditions 


\section{Near-Collision / Collision}

A near-collision was counted if the CG's of the two aircraft were $<300 \mathrm{ft}$ apart laterally and vertical separation was $<200 \mathrm{ft}$. A collision was counted if the aircraft CG's were $<150 \mathrm{ft}$ apart laterally and vertical separation was $<100 \mathrm{ft}$.

\section{Nuisance / Missed Indications and Alerts}

According to the SURF IA SPR [5], a nuisance indication or alert is defined as any indication or alert generated by a properly functioning CD\&R system that is inappropriate or unnecessary for the particular situation. Nuisance indications and alerts could distract the flight crew unnecessarily, reduce confidence in the system, and negatively affect safety and operational effectiveness. Repeated nuisance indications and alerts could decrease the use of $\mathrm{CD} \& \mathrm{R}$ and reduce expeditious flight crew response to true indications and alerts.

A missed indication or alert is defined as a failure to provide an indication or alert when it is necessary provided own-ship and traffic are adequately equipped [5]. Missed indications and alerts represent a reduction in $C D \& R$ benefits.

The SURF IA SPR [5] definitions for nuisance and missed boundaries were applied. Horizontal position error was the only source of error modeled. Other sources of error, such as vertical position error, airport database error and flight technical error, were not included.

The following nuisance and missed boundary definitions applied for Aircraft A:

- When the aircraft was on approach, an approach corridor as defined in the SURF IA SPR for NACp 8 with a probability of missed alert of 0.01 was used since NACp 8 and higher was being evaluated. The corridor width was +/$321.5 \mathrm{ft}$ at the runway threshold and linearly increased to +/- $964.6 \mathrm{ft}$ at $3 \mathrm{~nm}$ away from the runway threshold. The nuisance boundary definition was when the true aircraft position was outside the approach corridor, but the detected position was within the approach corridor. The missed boundary definition was when the aircraft's true position was within the approach corridor, but the detected position was outside the approach corridor. Since the true position of the approach aircraft tracked the extended runway centerline, the aircraft could never enter the nuisance boundary.

- When the aircraft had crossed the runway threshold on landing or was traveling along a runway, the nuisance boundary definition was when the aircraft's true position was farther than one runway width $(150 \mathrm{ft})$ from the runway centerline, but the detected position was within one runway width of the centerline. The missed boundary definition was when the aircraft's true position was within one runway width of the runway centerline, but the detected position was greater than one runway width from the centerline.

The following nuisance and missed boundary definitions applied for Aircraft B:

- When the aircraft was taxiing across the runway, the nuisance boundary definition was when the true position of the aircraft's nose (when entering) or tail (when exiting) was at or behind the hold line, but any part of the detected aircraft (from nose to tail) was between the runway shoulder edges. The missed boundary definition was when the true position of any part of the aircraft was between the runway shoulder edges, but the detected nose position (when entering) or tail position (when exiting) was outside of the runway shoulder edges. A shoulder width of 7.5 $\mathrm{m}(25 \mathrm{ft})$, as defined in the SURF IA SPR for Aerodrome Code 4, was used. A $150 \mathrm{ft}$ wide runway was assumed; therefore, the distance between shoulder edges was approximately 200 $\mathrm{ft}$. The hold line was located $225 \mathrm{ft}$ from the runway centerline.

Since only true vertical position was used, there was no opportunity for a nuisance or missed condition in the vertical direction.

An indication or alert was considered to be a nuisance if the indication or alert was generated when the aircraft was within a nuisance boundary, based on the definitions above.

A straight-forward corollary for a missed alert definition does not exist. If the aircraft was within the missed boundary, based on the definitions above, and an alert was not generated, that did not necessarily mean that an alert should have been generated. Even though one of the aircraft was in the missed boundary, the geometry of the aircrafts' trajectory 
may not be on a collision path. Therefore, if an alert was generated when using truth data but an alert was not generated at the same instance when using NACp data, then a missed alert was counted. This definition is algorithm dependent.

\section{Results}

A summary of quantitative results is presented. All data is referenced from the aircraft CG, unless noted otherwise. For the aircraft used in this study, the nose position was $72.8 \mathrm{ft}$ from the $\mathrm{CG}$ and the tail position was $82 \mathrm{ft}$ from the $\mathrm{CG}$.

For each scenario, the data analysis was limited to the area of interest, i.e., until the aircraft reached the CPA or until 10 seconds after a warning alert terminated, whichever was later. Also, both aircraft broadcasted the same level of positional accuracy for each test run.

The Kruskal-Wallis test was utilized to provide a non-parametric statistical hypothesis test to detect differences in NACp accuracy and CD\&R equipage using a significance level of $\alpha=0.05$ and $\mathrm{N}=$ number of test runs. When statistically significant differences were detected, post-hoc analysis was conducted using the non-parametric Mann-Whitney test.

\section{Runway Scenario - Arrival with taxi crossing}

For each of the 20 cases in this scenario, 2,367 combinations of the initiation delay and initial position for Aircraft B (taxiing aircraft) were evaluated, for a total of 198,828 test runs.

Algorithm performance - The data (Table 3) shows that, for Aircraft A, TIs were generated on $20 \%$ to $57 \%$ of the test runs and RSIs were issued on approximately $30 \%$ of runs. For Aircraft B, TIs were generated on $57 \%$ to $81 \%$ of runs and RSIs were issued on approximately $75 \%$ of runs. Caution alerts were generated on approximately $24 \%$ of the runs and warning alerts were issued on approximately $37 \%$ of the runs for either aircraft, almost independent of the NACp levels. The number of TIs for NACp 8 accuracy was statistically different from NACp 9, 10, 11, and truth accuracies over all equipage levels. There was no statistical difference between accuracy levels for the number of RSIs, caution, and warning alerts over all equipage levels.
Toggling occurred when multiple instances of indications and alerts were generated during a test run. Toggling is undesirable (i.e., it is a distraction to the flight crew and would cause mistrust in the technology).

Toggling occurred frequently for NACp 8 accuracy (see Table 3). The number of multiple TIs, RSIs, and caution and warning alerts for NACp 8 accuracy was statistically different from NACp 9, 10, 11 and truth accuracies over all equipage levels. This toggling included gaps between indications and alerts in many instances.

For Aircraft B, toggling occurred for indications when using true position data. In some instances this occurred by design. Some situations warrant a TI; as the situation progresses, an RSI or alert is generated, then, if the situation changes, the indication may be degraded back to a less severe TI. It was determined that some of the multiple indications occurred as Aircraft B taxied across an intersecting runway prior to crossing Runway 10. A TI was issued as the aircraft was taxiing toward Runway 10; however, before reaching Runway 10, the aircraft crossed an intersecting runway. Since indications are not required in that situation, the indication was no longer issued. After crossing the intersecting runway, however, the TI was issued again as the aircraft approached Runway 10. The SURF IA algorithm does not have any mechanisms in place to address toggling between aircraft states.

Missed and nuisance alerts - The number of runs in which the aircraft entered the defined missed and nuisance boundaries increased as the position accuracy decreased, as shown in Table 4. Aircraft can cross into the missed and nuisance boundary multiple times throughout a test run, for varying lengths of time. The number of times (count) and amount of time (duration and percentage of run length) that the aircraft were within the boundaries was generally greater when using less accurate data (see Table 4). However, there was no statistical difference between accuracy levels when analyzing the number of times entering the missed and nuisance boundary over all equipage levels.

For the approach aircraft (Aircraft A), the majority of occurrences of entering the missed boundary was after the aircraft had crossed the runway threshold for landing. The aircraft only entered the missed boundary while on approach 
Table 3. Indication and Alert Statistics by Aircraft for Arrival / Taxi Crossing Runway Scenario

\begin{tabular}{|c|c|c|c|c|c|c|c|c|c|}
\hline NACp & $\begin{array}{c}\text { Total \# } \\
\text { Runs }\end{array}$ & $\begin{array}{c}\text { TI } \\
\text { (\% runs) }\end{array}$ & $\begin{array}{c}\text { Multiple } \\
\text { TI } \\
\text { (\% runs) }\end{array}$ & $\begin{array}{c}\text { RSI } \\
\text { (\% runs) }\end{array}$ & $\begin{array}{c}\text { Multiple } \\
\text { RSI } \\
\text { (\% runs) }\end{array}$ & $\begin{array}{c}\text { Caution } \\
\text { Alerts } \\
\text { (\% runs) }\end{array}$ & $\begin{array}{c}\text { Multiple } \\
\text { Caution } \\
\text { Alerts } \\
\text { (\% runs) }\end{array}$ & $\begin{array}{c}\text { Warning } \\
\text { Alerts } \\
\text { (\% runs) }\end{array}$ & $\begin{array}{c}\text { Multiple } \\
\text { Warning } \\
\text { Alerts } \\
\text { (\% runs) }\end{array}$ \\
\hline \multicolumn{8}{|c|}{ Aircraft A } \\
\hline 8 & 66,276 & 57.0 & 17.6 & 32.5 & 5.8 & 26.0 & 2.5 & 33.4 & 9.8 \\
\hline 9 & 56,808 & 28.0 & 1.3 & 31.3 & 1.1 & 24.0 & $0.0 *$ & 37.4 & 1.8 \\
\hline 10 & 37,872 & 21.2 & 0.0 & 31.5 & 0.0 & 24.1 & 0.0 & 37.5 & 0.0 \\
\hline 11 & 28,404 & 19.4 & 0.0 & 29.0 & 0.0 & 24.0 & 0.0 & 37.3 & 0.0 \\
\hline Truth & 9,468 & 21.1 & 0.0 & 31.7 & 0.0 & 24.0 & 0.0 & 37.3 & 0.0 \\
\hline \multicolumn{7}{|c|}{ Aircraft B } & & & 11.6 \\
\hline 8 & 66,276 & 81.0 & 41.1 & 78.6 & 36.7 & 26.0 & 5.1 & 33.6 & 2.1 \\
\hline 9 & 56,808 & 67.9 & 13.1 & 79.5 & 11.0 & 24.0 & 0.1 & 37.6 & 0.0 \\
\hline 10 & 37,872 & 63.3 & 6.8 & 79.1 & 3.6 & 24.2 & 0.0 & 37.9 & 0.0 \\
\hline 11 & 28,404 & 57.2 & 6.2 & 72.5 & 2.7 & 24.2 & 0.0 & 37.8 & 0.0 \\
\hline Truth & 9,468 & 61.7 & 5.8 & 78.8 & 2.1 & 24.1 & 0.0 & 37.8 & 0.0 \\
\hline
\end{tabular}

* Only four occurrences

Table 4. Missed and Nuisance Boundary Statistics by Aircraft for Arrival / Taxi Crossing Runway Scenario

\begin{tabular}{|c|c|c|c|c|c|c|c|c|}
\hline \multirow[b]{2}{*}{ NACp } & \multicolumn{4}{|c|}{ Entered Missed Boundary } & \multicolumn{4}{|c|}{ Entered Nuisance Boundary } \\
\hline & $\begin{array}{l}\text { \# runs, } \\
\% \text { runs }\end{array}$ & $\begin{array}{c}\text { Count } \\
\text { (weighted } \\
\text { mean, SD) }\end{array}$ & $\begin{array}{c}\text { Duration } \\
\text { (seconds) } \\
\text { (mean, SD) }\end{array}$ & $\begin{array}{c}\% \text { of } \\
\text { Run } \\
\text { Length } \\
\end{array}$ & $\begin{array}{l}\text { \# runs, } \\
\text { \% runs }\end{array}$ & $\begin{array}{c}\text { Count } \\
\text { (weighted } \\
\text { mean, SD) }\end{array}$ & \begin{tabular}{|c}
$\begin{array}{c}\text { Duration } \\
\text { (seconds) } \\
\text { (mean, SD) }\end{array}$ \\
\end{tabular} & $\begin{array}{c}\% \text { of } \\
\text { Run } \\
\text { Length }\end{array}$ \\
\hline \multicolumn{9}{|c|}{ Aircraft A } \\
\hline 8 & $4669,82.5$ & $6.9,4.2$ & $11.7, \quad 11.9$ & 7.9 & $799, \quad 1.2$ & $\begin{array}{ll}1.0, & 1.0 \\
\end{array}$ & $0.6, \quad 0.2$ & 0.3 \\
\hline 9 & $744,1.3$ & $1.5, \quad 1.0$ & 1.5 & 0.8 & $395, \quad 0.7$ & $1.0, \quad 1.0$ & $\begin{array}{ll}0.6, & 0.2\end{array}$ & 0.2 \\
\hline 10 & $224,0.6$ & $1.0, \quad 0.0$ & 0.5 & 0.2 & $231,0.6$ & $1.0, \quad 0.0$ & $0.4, \quad 0.2$ & 0.2 \\
\hline 11 & $116,0.4$ & $1.0, \quad 0.0$ & 0.2 & 0.1 & $104, \quad 0.4$ & $1.0, \quad 0.0$ & $0.2, \quad 0.1$ & 0.1 \\
\hline Truth & $0,0.0$ & $0.0, \quad 0.0$ & 0.0 & 0.0 & $0,0.0$ & $0.0, \quad 0.0$ & $0.0, \quad 0.0$ & 0.0 \\
\hline \multicolumn{9}{|c|}{ Aircraft B } \\
\hline 8 & $54627,82.4$ & $2.1,1.6$ & $4.1,2.8$ & 3.6 & $19692,29.7$ & $\begin{array}{ll}3.6, & 1.7\end{array}$ & $6.7,10.6$ & 5.3 \\
\hline 9 & $42329,74.5$ & $1.3, \quad 1.1$ & $1.5, \quad 1.0$ & 1.3 & $58, \quad 0.1$ & $\begin{array}{ll}1.6, & 0.8 \\
\end{array}$ & $1.3, \quad 2.0$ & 1.2 \\
\hline 10 & $25011,66.0$ & $1.1, \quad 1.0$ & $0.6,0.3$ & 0.5 & $0, \quad 0.0$ & $0.0, \quad 0.0$ & $0.0, \quad 0.0$ & 0.0 \\
\hline 11 & $12223,43.0$ & $\begin{array}{ll}1.1, & 0.9\end{array}$ & $0.3,0.1$ & 0.2 & $0, \quad 0.0$ & $0.0, \quad 0.0$ & $0.0, \quad 0.0$ & 0.0 \\
\hline Truth & $0, \quad 0.0$ & $0.0, \quad 0.0$ & $0.0, \quad 0.0$ & 0.0 & $0, \quad 0.0$ & $0.0, \quad 0.0$ & $0.0, \quad 0.0$ & 0.0 \\
\hline
\end{tabular}

before crossing the runway threshold during $2.8 \%$ of the test runs when using NACp 8 accuracy. Since Aircraft A tracked the extended centerline on approach and centerline after landing, the nuisance boundary was entered as the aircraft was exiting the runway.

The taxiing aircraft (Aircraft B) entered the missed boundary at least once for a high percentage of the test runs for accuracy levels of NACp 8 to 11 .
This was due to the criteria for entering the missed boundary. The aircraft was counted as entering the missed boundary when the true position of any part of the aircraft was determined to be between the runway shoulder edges, but the detected nose position (when entering) or tail position (when exiting) was outside of the runway shoulder edges. There was no buffer between when the aircraft was inside or outside the missed boundary so measurable 
difference between the true and detected position could cause a missed boundary to be counted.

The number of test runs that contained missed and nuisance indications and alerts were relatively low, overall, as shown in Table 5. The missed indication and alert definition, as noted previously, is algorithm dependent. Missed indications and alerts for both aircraft were highest when using NACp 8 accuracy. There was no statistical difference between accuracy levels for the number of missed TIs, RSIs, and caution alerts over all equipage levels. The number of missed warning alerts for NACp 8 accuracy was statistically different from NACp 9, 10, 11 , and truth accuracies over all equipage levels. For Aircraft B only, the number of nuisance TIs, RSIs, and caution and warning alerts for NACp 8 accuracy was statistically different from NACp 9, 10, 11, and truth accuracies over all equipage levels.

Aircraft B did, however, experience missed TIs under the truth accuracy condition. This unexpected event occurred because even though the aircraft were broadcasting true position data, the ADS-B transmission model was still being used. The transmission model resulted in a slight delay between the aircraft's position at the time of transmitting the ADS-B message and the position at the time of reception of the ADS-B message by Aircraft $\mathrm{B}$. This delay was present in all scenarios, but this position difference was negligible compared to the NACp position uncertainty error. In the missed TI scenarios, Aircraft B did not detect a potential conflict with Aircraft A based on the broadcast position, but if instantaneous position information were used for Aircraft A, a potential conflict would have been detected. The small error introduced by the movement of Aircraft A between transmission and reception of the ADS-B message resulted in just enough difference in relation to Aircraft B's position to result in the missed alerts.

For Aircraft A, all of the missed indications and alerts, for all accuracy levels, occurred after the aircraft had crossed the runway threshold for landing. An indication was considered a nuisance if it was generated at the same time the aircraft was determined to be within the nuisance boundary. Therefore, nuisance indications for Aircraft A only occurred as the aircraft was exiting the runway (see explanation above).

Unnecessary maneuvering - Previous research has shown that pilots instinctively react upon receiving airport traffic warning alerts in the flight deck [13] without necessarily confirming with secondary or additional information first. It is critical that alerting only occurs when needed; otherwise,

Table 5. Missed and Nuisance Indication and Alert Statistics by Aircraft for Arrival / Taxi Crossing Runway Scenario

\begin{tabular}{|c|c|c|c|c|c|c|c|c|c|}
\hline \multirow{2}{*}{ NACp } & $\begin{array}{c}\text { Total \# } \\
\text { Runs }\end{array}$ & $\begin{array}{c}\text { TI } \\
\text { (\% runs) }\end{array}$ & $\begin{array}{c}\text { RSI } \\
\text { (\% runs) }\end{array}$ & $\begin{array}{c}\text { Caution } \\
\text { Alert } \\
\text { \% runs) }\end{array}$ & $\begin{array}{c}\text { Warning } \\
\text { Alert } \\
\text { (\% runs) }\end{array}$ & $\begin{array}{c}\text { TI } \\
\text { (\% runs) }\end{array}$ & $\begin{array}{c}\text { RSI } \\
\text { (\% runs) }\end{array}$ & $\begin{array}{c}\text { Caution } \\
\text { Alert } \\
\text { (\% runs) }\end{array}$ & $\begin{array}{c}\text { Warning } \\
\text { Alert } \\
\text { (\% runs) }\end{array}$ \\
\hline \multicolumn{8}{|c|}{ Aircraft A } \\
\hline 8 & 66,276 & 0.5 & 1.8 & 3.2 & 6.8 & $0.0^{*}$ & 0.0 & 0.0 & 0.0 \\
\hline 9 & 56,808 & 0.1 & 0.3 & 1.2 & 0.8 & $0.0^{*}$ & 0.0 & 0.0 & 0.0 \\
\hline 10 & 37,872 & $0.0^{*}$ & 0.1 & 0.3 & 0.1 & 0.0 & 0.0 & 0.0 & 0.0 \\
\hline 11 & 28,404 & 0.0 & $0.0^{*}$ & 0.1 & $0.0+$ & 0.0 & 0.0 & 0.0 & 0.0 \\
\hline Truth & 9,468 & 0.0 & 0.0 & 0.0 & 0.0 & 0.0 & 0.0 & 0.0 & 0.0 \\
\hline \multicolumn{7}{|c|}{ Aircraft B } & & & \\
\hline 8 & 66,276 & 1.4 & 3.2 & 3.2 & 6.8 & 6.1 & 5.6 & 4.4 & 4.1 \\
\hline 9 & 56,808 & 0.7 & 0.6 & 1.2 & 0.8 & 0.0 & 0.0 & 0.0 & 0.0 \\
\hline 10 & 37,872 & 0.4 & 0.2 & 0.3 & 0.1 & 0.0 & 0.0 & 0.0 & 0.0 \\
\hline 11 & 28,404 & 0.2 & $0.0^{*}$ & 0.1 & $0.0+$ & 0.0 & 0.0 & 0.0 & 0.0 \\
\hline Truth & 9,468 & 0.1 & 0.0 & 0.0 & 0.0 & 0.0 & 0.0 & 0.0 & 0.0 \\
\hline
\end{tabular}

* Negligible number of occurrences

+12 occurrences 
these unnecessary maneuvers can cause delays, equipment wear, and other costs to airlines.

To evaluate this situation, maneuvering was considered unnecessary if made based on a warning alert issued when the aircraft were broadcasting NACp accuracy, but for the same test conditions, a warning alert was not issued when broadcasting true position data. This measure quantifies untimely nuisance alerts using an algorithm-dependent methodology.

The percentage of test runs in which the aircraft maneuvered unnecessarily when using NACp data accuracy is shown in Table 6. Thus, as the accuracy decreased, the frequency of occurrences of unnecessary maneuvers increased. With a NACp value of 8 , approximately $7 \%$ or 7 in 100 of the warning alerts were unnecessary. With a NACp value of 11, approximately 1 in 1000 warning alerts were unnecessary for Aircraft $A$ and 5 in 1000 were unnecessary for Aircraft B. The number of unnecessary maneuvers for NACp 8 accuracy was statistically different from NACp 9, 10, and 11 accuracies for both Aircraft A and Aircraft B.

Table 6. Unnecessary Maneuvers by Aircraft during Arrival / Taxi Crossing Runway Scenario

\begin{tabular}{|c|c|c|}
\hline NACp & $\begin{array}{c}\text { Aircraft A } \\
\text { (\% runs) }\end{array}$ & $\begin{array}{c}\text { Aircraft B } \\
\text { (\% runs) }\end{array}$ \\
\hline 8 & 7.4 & 7.5 \\
\hline 9 & 1.2 & 1.4 \\
\hline 10 & 0.5 & 0.9 \\
\hline 11 & 0.1 & 0.5 \\
\hline
\end{tabular}

Collision avoidance - By the design of the scenarios, approximately $20 \%$ of the runs resulted in a near collision (NC) and approximately $10 \%$ resulted in a collision (C) in the absence of CD\&R, as shown in Table 7. The addition of CD\&R did not have much effect on collision avoidance with approximately $18 \%$ of the runs resulting in near collision and approximately $8 \%$ in collisions when both were equipped. Collision avoidance was not significantly affected by the CD\&R system equipage levels.

For the taxiing aircraft (Aircraft B), a warning alert was not timely. The warning generally did not occur until the aircraft was on or almost on the runway (i.e., the mean, true position of Aircraft B when it received the warning alert was $107.6 \mathrm{ft}$, standard deviation (SD) $59.9 \mathrm{ft}$, from the runway centerline). Since the aircraft was already past the runway shoulder, no action was taken and the aircraft continued across the runway (as per the design of the test maneuvers).

Some collisions were also unavoidable since the approach aircraft (Aircraft A) was issued a warning alert during a critical operational phase. Most often, the warning alert occurred during high speed rollout without enough separation from the traffic to stop in time to avoid a collision. Other times the warning occurred when the aircraft was too close to the ground to go around and the collision occurred as the aircraft continued along its predetermined path. Some collisions also occurred during low speed ( $>40$ kt) taxi on the runway or as the aircraft was exiting the runway - conditions for which the SURF IA $\mathrm{CD} \& \mathrm{R}$ algorithm does not issue warning alerts; therefore, no avoidance action was taken.

Collision avoidance was not significantly affected by the horizontal position accuracy level; however, there were slightly less collisions when using NACp 8 accuracy. In some instances, the warning alert was generated when the sensed aircraft was on the runway, but the actual (true) location of the aircraft was far enough back from the runway that it could come to a complete stop before actually reaching the runway shoulder.

\section{Runway Scenario - Departure with taxi crossing}

For each of the 20 cases in this scenario, 1,077 combinations of the initiation delay and initial position for Aircraft B were evaluated, for a total of 90,468 test runs.

Algorithm performance - The data (Table 8) shows that, for Aircraft A, TIs were generated on $0 \%$ to $50 \%$ of the test runs and RSIs were issued on approximately $3 \%$ or less of runs. For Aircraft B, TIs were generated on $60 \%$ to $87 \%$ of runs and RSIs were issued on approximately $77 \%$ of runs. Warning alerts were issued on approximately $30 \%$ of the runs for either aircraft, almost independent of the NACp levels. Caution alerts were not generated by either aircraft for this scenario. The number of TIs for NACp 8 accuracy was statistically different from NACp 9, 10, 11, and truth accuracies over all 
Table 7. Percentage of Near-Collisions (NC) and Collisions (C) for Equipage Combinations for Arrival / Taxi Crossing Runway Scenario

\begin{tabular}{|l|c|c|c|c|c|c|c|c|c|}
\hline \multirow{2}{*}{ NACp } & \# Runs per & \multicolumn{2}{|c|}{ Neither } & \multicolumn{2}{c|}{ Aircraft A } & \multicolumn{2}{c|}{ Aircraft B } & \multicolumn{2}{c|}{ Both } \\
\cline { 3 - 9 } & Equipage & NC & C & NC & C & NC & C & NC & C \\
\hline 8 & 16,569 & 19.3 & 9.3 & 18.7 & 8.9 & 18.9 & 7.9 & 17.9 & 8.0 \\
\hline 9 & 14,202 & 19.3 & 9.3 & 19.0 & 9.2 & 18.7 & 9.0 & 18.5 & 8.7 \\
\hline 10 & 9,468 & 19.3 & 9.3 & 19.0 & 9.2 & 18.6 & 8.9 & 18.4 & 8.8 \\
\hline 11 & 7,101 & 19.3 & 9.3 & 19.0 & 9.0 & 18.7 & 8.9 & 18.4 & 8.8 \\
\hline Truth & 2,367 & 19.3 & 9.3 & 19.1 & 9.1 & 18.6 & 9.0 & 18.4 & 8.7 \\
\hline
\end{tabular}

Table 8. Indication and Alert Statistics by Aircraft for Departure / Taxi Crossing Runway Scenario

\begin{tabular}{|c|c|c|c|c|c|c|c|}
\hline NACp & $\begin{array}{c}\text { Total \# } \\
\text { Runs }\end{array}$ & $\begin{array}{c}\text { TI } \\
\text { (\% runs) }\end{array}$ & $\begin{array}{c}\text { Multiple TI } \\
\text { (\% runs) }\end{array}$ & $\begin{array}{c}\text { RSI } \\
\text { ( runs) }\end{array}$ & $\begin{array}{c}\text { Multiple RSI } \\
\text { (\% runs) }\end{array}$ & $\begin{array}{c}\text { Warning Alerts } \\
\text { (\% runs) }\end{array}$ & $\begin{array}{c}\text { Multiple Warning } \\
\text { Alerts } \\
\text { (\% runs) }\end{array}$ \\
\hline \multicolumn{7}{|c|}{ Aircraft A } \\
\hline 8 & 30,156 & 49.8 & 14.4 & 3.3 & 1.0 & 24.9 & 6.3 \\
\hline 9 & 25,848 & 7.4 & 1.0 & 1.1 & 0.1 & 31.1 & 1.1 \\
\hline 10 & 17,232 & 0.0 & 0.0 & 0.6 & 0.0 & 31.7 & 0.0 \\
\hline 11 & 12,924 & 0.0 & 0.0 & 0.7 & 0.0 & 31.8 & 0.0 \\
\hline Truth & 4,308 & 0.0 & 0.0 & 0.7 & 0.0 & 31.7 & 0.0 \\
\hline \multicolumn{7}{|c|}{ Aircraft B } \\
\hline 8 & 30,156 & 87.1 & 70.7 & 66.0 & 34.9 & 24.3 & 7.5 \\
\hline 9 & 25,848 & 67.8 & 14.2 & 77.8 & 9.2 & 30.2 & 1.0 \\
\hline 10 & 17,232 & 61.2 & 0.8 & 77.8 & 0.6 & 30.8 & 0.0 \\
\hline 11 & 12,924 & 60.1 & 0.8 & 77.7 & 0.1 & 30.9 & 0.0 \\
\hline Truth & 4,308 & 59.9 & 0.7 & 77.7 & 0.0 & 30.7 & 0.0 \\
\hline
\end{tabular}

equipage levels. There was no statistical difference between accuracy levels for the number of RSIs and warning alerts over all equipage levels.

As the position accuracy was reduced, indication and alert toggling occurred more frequently, particularly for NACp 8 and 9 accuracies (see Table 8 ). For Aircraft A, the number of multiple TIs and warning alerts for NACp 8 accuracy was statistically different from NACp 9, 10, 11, and truth accuracies over all equipage levels. There was no statistical difference between accuracy levels for number of multiple RSIs over all equipage levels. For Aircraft $\mathrm{B}$, the number of multiple TIs and RSIs for NACp 8 accuracy was statistically different from NACp 9 and NACp 9 was statistically different from NACp 10, 11 , and truth accuracies over all equipage levels. The number of multiple warning alerts for NACp 8 accuracy was statistically different from NACp 9, 10,
11 and truth accuracies over all equipage levels. The toggling included gaps between alerts in many instances. In addition to position accuracy, the toggling can also be a result of aircraft collision avoidance maneuvering.

For Aircraft B, TI toggling occurred when accurate (true) position data was transmitted. This toggling occurred by design. A TI was initially issued for Aircraft B and as the situation progressed, an RSI and warning alert were issued, then, after Aircraft A rejected the departure, a TI was issued again.

The SURF IA SPR [5] specifies that indications and alerts must be inhibited above $80 \mathrm{kt}$. But as currently implemented, the SURF IA algorithm calculates alerts throughout the departure when the aircraft is traveling greater than $80 \mathrm{kt}$ (the mean ground speed when a warning alert was generated on 
Aircraft A was $121.7 \mathrm{kt}$, SD 36.6 for truth accuracy). These data were included in Table 8 even though these alerts would not be displayed in the cockpit as per the SPR. For the test runs in which the departing aircraft was to take action (Aircraft A only and both equipped), warning alerts were generated when the aircraft was traveling less than $80 \mathrm{kt}$ in only $5 \%$ to $7 \%$ of the test runs; without this restriction, warning alerts were generated on $23 \%$ to $32 \%$ of the test runs (see Table 9). More research is necessary to determine the collision avoidance benefits of alerting after $80 \mathrm{kt}$ versus the risk of pilots making inappropriate responses at high speed.

\section{Table 9. Warning Alert Data for Departure} Aircraft

\begin{tabular}{|c|c|c|}
\hline NACp & $\begin{array}{c}\text { Warning Alerts } \\
\text { (\% runs) }\end{array}$ & $\begin{array}{c}\text { Warning Alerts } \\
\text { when }<80 \mathrm{kt} \\
\text { (\% runs) }\end{array}$ \\
\hline 8 & 23.4 & 7.0 \\
\hline 9 & 32.5 & 5.9 \\
\hline 10 & 31.7 & 5.2 \\
\hline 11 & 31.8 & 5.3 \\
\hline Truth & 29.6 & 5.2 \\
\hline
\end{tabular}

Missed and nuisance alerts - The number of runs in which the aircraft entered the defined missed and nuisance boundaries increased as the position accuracy decreased, as shown in Table 10. The number of times (count) and amount of time (duration and percentage of run length) that the aircraft were within the boundaries was greater when using less accurate data (see Table 10). This was particularly true when using NACp 8 accuracy. The number of times Aircraft $\mathrm{A}$ entered the missed boundary for NACp 8 accuracy was statistically different from NACp 9, 10, 11, and truth accuracies over all equipage levels. There were no instances of Aircraft A entering the nuisance boundary. For Aircraft B, there was no statistical difference between NACp accuracy levels when analyzing the number of times entering the missed boundary over all equipage levels. The number of times Aircraft B entered the nuisance boundary for NACp 8 accuracy was statistically different from NACp 9, 10, 11, and truth accuracies over all equipage levels.

The departing aircraft (Aircraft A) entered the missed boundary along its entire departure path. Since Aircraft A tracked the runway centerline on departure, it was not possible for the aircraft to enter the nuisance boundary.

The taxiing aircraft (Aircraft B) entered the missed boundary at least once for a high percentage of the test runs for accuracy levels of NACp 8 to 11 . This was due to the criteria for entering the missed boundary. The aircraft was counted as entering the missed boundary when the true position of any part of the aircraft was determined to be between the runway shoulder edges, but the detected nose position (when entering) or tail position (when

Table 10. Missed and Nuisance Boundary Statistics by Aircraft for Departure / Taxi Crossing Runway Scenario

\begin{tabular}{|c|c|c|c|c|c|c|c|c|}
\hline \multirow[b]{2}{*}{ NACp } & \multicolumn{4}{|c|}{ Entered Missed Boundary } & \multicolumn{4}{|c|}{ Entered Nuisance Boundary } \\
\hline & $\begin{array}{l}\text { \# runs, } \\
\% \text { runs }\end{array}$ & $\begin{array}{c}\text { Count } \\
\text { (weighted } \\
\text { mean, SD) }\end{array}$ & $\begin{array}{c}\begin{array}{c}\text { Duration } \\
\text { (seconds) } \\
\text { (mean, SD) }\end{array} \\
\end{array}$ & \begin{tabular}{|c|}
$\%$ of \\
Run \\
Length \\
\end{tabular} & $\begin{array}{l}\text { \# runs, } \\
\text { \% runs }\end{array}$ & $\begin{array}{c}\text { Count } \\
\text { (weighted } \\
\text { mean, SD) }\end{array}$ & $\begin{array}{c}\begin{array}{c}\text { Duration } \\
\text { (seconds) } \\
\text { (mean, SD) }\end{array} \\
\end{array}$ & $\begin{array}{c}\% \text { of } \\
\text { Run } \\
\text { Length }\end{array}$ \\
\hline \multicolumn{9}{|c|}{ Aircraft A } \\
\hline 8 & $24675,81.8$ & $4.4, \quad 3.5$ & 9.0 & 23.7 & $0, \quad 0.0$ & $0.0, \quad 0.0$ & $0.0, \quad 0.0$ & 0.0 \\
\hline 9 & $48,0.2$ & $2.1, \quad 1.2$ & 2.1 & 5.0 & $0,0.0$ & $0.0, \quad 0.0$ & $0.0, \quad 0.0$ & 0.0 \\
\hline 10 & $0,0.0$ & $0.0, \quad 0.0$ & 0.0 & 0.0 & $0, \quad 0.0$ & $0.0, \quad 0.0$ & $0.0, \quad 0.0$ & 0.0 \\
\hline 11 & $0,0.0$ & $0.0, \quad 0.0$ & 0.0 & 0.0 & $0, \quad 0.0$ & $0.0, \quad 0.0$ & $0.0, \quad 0.0$ & 0.0 \\
\hline Truth & $0,0.0$ & $0.0, \quad 0.0$ & 0.0 & 0.0 & $0, \quad 0.0$ & $0.0, \quad 0.0$ & $0.0, \quad 0.0$ & 0.0 \\
\hline \multicolumn{9}{|c|}{ Aircraft B } \\
\hline 8 & $18392,61.0$ & $1.6, \quad 1.2$ & 3.6 & 9.6 & 5946, 19.7 & $2.4, \quad 1.4$ & $\begin{array}{ll}4.2, & 7.8\end{array}$ & 9.2 \\
\hline 9 & $11479,44.4$ & $1.1, \quad 1.0$ & 1.3 & 3.8 & $8, \quad 0.1$ & $1.1, \quad 0.8$ & $0.3, \quad 0.2$ & 0.9 \\
\hline 10 & $6419,37.3$ & $1.0,1.0$ & 0.5 & 1.5 & $0, \quad 0.0$ & $0.0, \quad 0.0$ & $0, \quad 0.0$ & 0.0 \\
\hline 11 & $2776,21.5$ & $1.0, \quad 1.0$ & 0.3 , & 0.8 & $0, \quad 0.0$ & $0.0, \quad 0.0$ & $0, \quad 0.0$ & 0.0 \\
\hline Truth & $0, \quad 0.0$ & $0.0, \quad 0.0$ & 0.0 & 0.0 & $0, \quad 0.0$ & $0.0, \quad 0.0$ & $0, \quad 0.0$ & 0.0 \\
\hline
\end{tabular}


Table 11. Missed and Nuisance Indication and Alert Statistics by Aircraft for Departure / Taxi Crossing Runway Scenario

\begin{tabular}{|c|c|c|c|c|c|c|c|c|c|}
\hline \multirow[b]{2}{*}{ NACp } & \multirow[b]{2}{*}{$\begin{array}{c}\text { Total \# } \\
\text { Runs }\end{array}$} & \multicolumn{4}{|c|}{ Missed } & \multicolumn{4}{|c|}{ Nuisance } \\
\hline & & $\begin{array}{c}\mathrm{TI} \\
(\% \text { runs })\end{array}$ & $\begin{array}{c}\text { RSI } \\
\text { (\% runs) }\end{array}$ & $\begin{array}{c}\text { Caution } \\
\text { Alert } \\
\text { (\% runs) }\end{array}$ & $\begin{array}{c}\text { Warning } \\
\text { Alert } \\
\text { (\% runs) }\end{array}$ & $\begin{array}{c}\mathrm{TI} \\
(\% \text { runs })\end{array}$ & $\begin{array}{c}\text { RSI } \\
\text { (\% runs) }\end{array}$ & $\begin{array}{c}\text { Caution } \\
\text { Alert } \\
\text { (\% runs) }\end{array}$ & $\begin{array}{c}\text { Warning } \\
\text { Alert } \\
\text { (\% runs) }\end{array}$ \\
\hline \multicolumn{10}{|c|}{ Aircraft A } \\
\hline 8 & 30,156 & 0.0 & 0.1 & 0.0 & 10.5 & 0.0 & 0.0 & 0.0 & 0.0 \\
\hline 9 & 25,848 & 0.0 & 0.0 & 0.0 & 1.9 & 0.0 & 0.0 & 0.0 & 0.0 \\
\hline 10 & 17,232 & 0.0 & 0.0 & 0.0 & 0.5 & 0.0 & 0.0 & 0.0 & 0.0 \\
\hline 11 & 12,924 & 0.0 & 0.0 & 0.0 & 0.2 & 0.0 & 0.0 & 0.0 & 0.0 \\
\hline Truth & 4,308 & 0.0 & 0.0 & 0.0 & 0.0 & 0.0 & 0.0 & 0.0 & 0.0 \\
\hline \multicolumn{10}{|c|}{ Aircraft B } \\
\hline 8 & 30,156 & 0.3 & 15.8 & 0.0 & 10.5 & 4.0 & 3.5 & 0.0 & 4.3 \\
\hline 9 & 25,848 & 0.5 & 2.4 & 0.0 & 1.9 & 0.0 & 0.0 & 0.0 & 0.0 \\
\hline 10 & 17,232 & 0.3 & 1.2 & 0.0 & 0.5 & 0.0 & 0.0 & 0.0 & 0.0 \\
\hline 11 & 12,924 & 0.1 & 0.9 & 0.0 & 0.2 & 0.0 & 0.0 & 0.0 & 0.0 \\
\hline Truth & 4,308 & 0.0 & 0.6 & 0.0 & 0.0 & 0.0 & 0.0 & 0.0 & 0.0 \\
\hline
\end{tabular}

exiting) was outside of the runway shoulder edges. As such, there was no buffer between when the aircraft was inside or outside the missed boundary so a measurable difference between the true and detected position could cause a missed boundary to be counted.

The number of test runs that contained missed and nuisance indications and alerts were relatively low, overall, as shown in Table 11. The missed indication and alert definition, as noted previously, is algorithm dependent. Missed indications and alerts for both aircraft were highest when using NACp 8 accuracy. For Aircraft A, only the number of missed warning alerts for NACp 8 accuracy was statistically different from NACp 9, 10, 11, and truth accuracies over all equipage levels. For Aircraft B, the number of nuisance TIs, missed and nuisance RSIs, and missed and nuisance warning alerts for NACp 8 accuracy was statistically different from NACp 9, 10, 11 , and truth accuracies over all equipage levels.

Aircraft B experienced missed RSIs under the truth accuracy condition. This was due to the transmission delay of the ADS-B model as described in the previous scenario.

Nuisance indications and alerts were not issued on Aircraft A because the aircraft tracked the runway centerline during departure and liftoff.
Unnecessary maneuvering - For the test runs in which both aircraft were broadcasting NACp accuracies and required to maneuver based on a warning alert, the percentage of test runs in which the aircraft maneuvered unnecessarily is shown in Table 12. As the accuracy decreased, the frequency of occurrences of unnecessary maneuvers increased. With a NACp value of 8 , approximately $8 \%$ or 8 in 100 of the warning alerts were unnecessary. With a NACp value of 11, approximately 2 in 1000 warning alerts were unnecessary. The number of unnecessary maneuvers for NACp 8 accuracy was statistically different from NACp 9, 10, 11, and truth accuracies for both Aircraft A and Aircraft B.

\section{Table 12. Unnecessary Maneuvers by Aircraft during Departure / Taxi Crossing Runway} Scenario

\begin{tabular}{|c|c|c|}
\hline NACp & $\begin{array}{c}\text { Aircraft A } \\
\text { (\% runs) }\end{array}$ & $\begin{array}{c}\text { Aircraft B } \\
\text { (\% runs) }\end{array}$ \\
\hline 8 & 8.5 & 8.0 \\
\hline 9 & 1.7 & 1.4 \\
\hline 10 & 0.5 & 0.3 \\
\hline 11 & 0.2 & 0.2 \\
\hline
\end{tabular}

Collision avoidance - By the design of the scenarios, approximately $14 \%$ of the runs resulted in a near collision and approximately $6 \%$ resulted in a collision in the absence of CD\&R, as shown in Table 
Table 13. Percentage of Near-Collisions (NC) and Collisions (C) for Equipage Combinations for Departure / Taxi Crossing Runway Scenario

\begin{tabular}{|l|c|c|c|c|c|c|c|c|c|}
\hline \multirow{2}{*}{ NACp } & \# Runs per & \multicolumn{2}{|c|}{ Neither } & \multicolumn{2}{c|}{ Aircraft A } & \multicolumn{2}{c|}{ Aircraft B } & \multicolumn{2}{c|}{ Both } \\
\cline { 3 - 10 } & Equipage & NC & C & NC & C & NC & C & NC & C \\
\hline 8 & 7,539 & 14.2 & 6.6 & 12.7 & 5.7 & 13.8 & 5.8 & 12.5 & 5.3 \\
\hline 9 & 6,462 & 14.2 & 6.6 & 12.6 & 5.8 & 14.1 & 6.4 & 12.6 & 5.7 \\
\hline 10 & 4,308 & 14.2 & 6.6 & 12.6 & 5.8 & 14.0 & 6.4 & 12.6 & 5.7 \\
\hline 11 & 3,231 & 14.2 & 6.6 & 12.6 & 5.7 & 14.0 & 6.4 & 12.5 & 5.7 \\
\hline Truth & 1,077 & 14.2 & 6.5 & 12.6 & 5.7 & 14.0 & 6.4 & 12.6 & 5.7 \\
\hline
\end{tabular}

13. The addition of CD\&R only slightly improved collision avoidance with approximately $12 \%$ of the runs resulting in a near collision and approximately $5 \%$ in a collision when both were equipped. Collision avoidance was not significantly affected by the $\mathrm{CD} \& \mathrm{R}$ system equipage levels.

For the taxiing aircraft (Aircraft B), a warning alert generally did not occur until the aircraft was on the runway (i.e., mean position of Aircraft B was $123.6 \mathrm{ft}$, SD 24.4, from the runway centerline when using truth accuracy). Since the aircraft was already past the runway shoulder, no action was taken and the aircraft continued across the runway.

For the departing aircraft (Aircraft A) when using true position accuracy, $16.6 \%$ of the warning alerts occurred during takeoff roll when traveling between 50 and $80 \mathrm{kt}, 41.9 \%$ occurred when traveling between 80 and $131 \mathrm{kt}$, and $41.5 \%$ occurred when traveling greater than $131 \mathrm{kt}$. The aircraft was unable to abort the departure after reaching takeoff decision speed $(131 \mathrm{kt})$; therefore, for $41.5 \%$ of the departures in which warning alerts were issued, the aircraft continued along its predefined departure path and collisions were unavoidable. As described above, alerts were not inhibited above $80 \mathrm{kt}$ as specified in the SURF IA SPR.

Position accuracy had little effect on collision avoidance; however, there were fewer collisions when Aircraft B was equipped when using NACp 8 accuracy. As in the previous scenario, this was because, in some instances, Aircraft B was able to conduct emergency braking and stop before reaching the runway shoulder. Even though the warning alert was generated when the aircraft was already on the runway (according to the data), the actual location of the aircraft was far enough back from the runway so it could come to a complete stop before actually reaching the runway shoulder. Over all levels of NACp accuracy, the differences in the number of collisions showed no statistically significant difference.

\section{Summary}

The SURF IA CD\&R algorithm was evaluated in a fast-time batch simulation study. The purpose of the study was to evaluate the performance of the aircraft-based SURF IA algorithm during various runway scenarios, multiple levels of CD\&R system equipage, and various levels horizontal position accuracy.

It is critical that alerting does not cause the flight crew to make unnecessary maneuvers since these actions can cause delays, equipment wear, and other costs to airlines. Results showed that for the scenarios reported on herein, maneuvers were frequently made unnecessarily when using NACp 8 position accuracy.

Alert toggling occurs when multiple instances of indications or alerts are generated as a result of position accuracy or aircraft maneuvering. Alert toggling can be a distraction to the flight crew and could cause mistrust in the technology. In general, alert toggling occurred more frequently as the position accuracy was reduced, especially for NACp 8 and NACp 9 accuracy levels.

Missed indications and alerts represent a reduction in $C D \& R$ benefits and will result in operations such as they currently exist, where CD\&R indications and alerts are not provided. The occurrence of missed indications and alerts was much higher with NACp 8 position accuracy, in general. 
Nuisance indications and alerts could distract the flight crew unnecessarily, reduce confidence in the system, and can negatively affect safety and operational effectiveness. For the test runs in which nuisance indications and alerts were possible, nuisance indications and alerts only occurred when using NACp 8 position accuracy.

For the scenarios reported on herein, the CD\&R equipage level did not have a major impact on collision avoidance. More collisions were avoided if both aircraft were equipped with $\mathrm{CD} \& \mathrm{R}$, but generally, the aircraft taxing across the runway was not issued a warning alert until the aircraft was on the runway and, therefore, did not take action and continued across the runway. Positional accuracy had surprisingly less effect on collision avoidance effectiveness than previously assumed. Continued analysis is necessary to understand if the ineffective $C D \& R$ was due to a test set-up assumption or is, in fact, a design fault of the present SURF IA SPR. Also, analysis is required to better understand the SPR $80 \mathrm{kt}$ restriction requirement for alerting during departure, the impact of not inhibiting alerts above 80 $\mathrm{kt}$ during departure, and the trade-off between missed alerts and increased nuisance/unnecessary alerting.

Continued analysis of these data is being conducted to identify horizontal positional accuracy requirements for effective terminal maneuvering area $\mathrm{CD \& R}$ and efficient algorithm designs.

\section{References}

[1] Federal Aviation Administration, 2011, ATO Safety, National Runway Safety Plan, V 1.0.

[2] Federal Aviation Administration, 2009, Annual Runway Safety Report 2009, Air Traffic Organization.

[3] National Transportation Safety Board, 2011, NTSB Most Wanted List Transportation Safety Improvements, www.ntsb.gov/safety/mwl.html

[4] National Transportation Safety Board, 2000, Safety Recommendation, Letter to the FAA Administrator, A-00-66.

[5] RTCA, Inc., 2010, Safety, Performance and Interoperability Requirements Document for Enhanced Traffic Situational Awareness on the Airport Surface with Indications and Alerts (SURF IA), DO-323.
[6] Moertl, P. M. and K. McGarry, 2011, Using a Cockpit Display of Traffic Information with Indications and Alerts to Prevent Runway Incursions, MITRE Case \# 11-0965.

[7] McGarry, K. and J. Helleberg, 2011, Using a CDTI with Indications to Prevent Runway Incursions, Proceedings of the International Symposium on Aviation Psychology.

[8] Moertl, P. M. and K. McGarry, 2011, Results of a Human in the Loop Simulation Investigating Display Requirements and Alerting Modalities of a Flight Deck Based Runway Safety Alerting System, MITRE Technical Report 110215.

[9] Bussink, F. J. L., J. Hoekstra, W. Heesbeen, 2005, Traffic Manager: A Flexible Desktop Simulation Tool Enabling Future ATM Research, Proceedings of the AIAA/IEEE $24^{\text {th }}$ Digital Avionics Systems Conference.

[10] RTCA, Inc., 2002, Minimum Aviation System Performance Standards for Automatic Dependent Surveillance Broadcast (ADS-B), DO-242A.

[11] Mohleji, S. C., G. Wang, 2010, Modeling ADS-B Position and Velocity Errors for Airborne Merging and Spacing in Interval Management Application, MITRE release \# 10-3026.

[12] Department of Transportation Federal Aviation Administration, 2010, Automatic Dependent Surveillance - Broadcast (ADS-B) Out Performance Requirements to Support Air Traffic Control (ATC) Service; Final Rule, Docket No. FAA-2007-29305; Amdt. No. 91-314.

[13] Jones, D. R., L. J. Prinzel, K. J. Shelton, R. E. Bailey, S. D. Otero, and G. D. Barker, 2010, Collision Avoidance for Airport Traffic Simulation Evaluation, Proceedings of the AIAA/IEEE $29^{\text {th }}$ Digital Avionics Systems Conference.

\section{Acknowledgements}

The authors would like to express their appreciation to the Air Traffic Operations Laboratory and Aeronautics Systems Engineering Branch personnel for their support throughout all phases of implementation, testing, and analysis.

\section{1st Digital Avionics Systems Conference October 14-18, 2012}


